\title{
Poleable Nanoparticles as Fillers Towards Non-linear Optically Active Actuators
}

\author{
Yee Song Ko ${ }^{\text {ab }}$, Frank A. Nüesch ${ }^{\text {ab }}$, Dorina M. Opris*a \\ ${ }^{a}$ Swiss Federal Laboratories for Materials Science and Technology Empa, Laboratory for Functional \\ Polymers, Überlandstr. 129, CH-8600, Dübendorf, Switzerland; ${ }^{b}$ Ecole Polytechnique Federale de \\ Lausanne (EPFL), Institut des materiaux, Station 12, CH 1015, Lausanne, Switzerland \\ *dorina.opris@empa.ch; phone +41 58765 4304; empa.ch
}

\begin{abstract}
A new type of poleable dielectric elastomer is introduced herein. The elastomer contains polymer nanoparticles with frozen molecular dipoles, which can be oriented at elevated temperatures in an electric field via poling. The aim is to provide a soft material with high, tunable optical properties suitable for actuator and flexible electronics applications. To that end poleable polymeric nanoparticles with high dipole concentrations and glass transition temperatures well above room temperature will be needed to be used as filler in an elastomer matrix. The synthesis and characterization of such particles is presented in this manuscript. Polyhydroxyethyl methacrylate (PHEMA) nanoparticles were synthesized using miniemulsion polymerization. The particles were loaded with 4-[ethyl (2-hydroxyethyl) amino]-4-nitrobenzene, usually called Disperse Red 1 (DR1), which has a large dipole moment $(\mu=7.5-9.5 \mathrm{D}){ }^{1}$ The maximum dipole loadings is limited by the solubility of the dipole in the monomer solutions prior to polymerization. All samples show a glass transition temperature around $95{ }^{\circ} \mathrm{C}$. Secondary electron microscopy (SEM) revealed spherical particles, the size of which was confirmed by dynamic light scattering (DLS) measurements. A composite was prepared by dispersing the particles in polydimethyl siloxane (PDMS).
\end{abstract}

Keywords: poling, dipole, PHEMA, DR1, miniemulsion polymerization, polymeric nanoparticles, permittivity, PHEMA-co-DR1.

\section{INTRODUCTION}

Dielectric elastomer actuators (DEAs) are a new type of electromechanical transducers based on the ability of an elastic capacitor to change dimensions when an electric field is applied. ${ }^{2,3,4}$ Linear actuators with output properties similar to that of biological muscle could have an immense impact in areas such as industrial and personal robotics, orthopaedic assists and medical devices in general. ${ }^{3}$ DEAs consist of an elastic film sandwiched between two compliant electrodes. ${ }^{5}$ When an electric voltage is applied, positive and negative charges are transferred onto the electrode surfaces inducing an electrostatic pressure which compresses and elongates the film, converting electrical energy into mechanical work. The electrostatic pressure $(p)$ acting on the film and the strain in thickness $\left(s_{z}\right)$ direction are given by: ${ }^{2 \mathrm{c}} p=\varepsilon \varepsilon_{0}\left(\frac{U}{d}\right)^{2}$ and $\mathrm{s}_{\mathrm{z}}$ $=\frac{p}{K}$, where $\varepsilon$ is the relative permittivity of the film, $\varepsilon_{0}$ the permittivity of free space, $U$ the applied voltage, $d$ the thickness of the film and $K$ the compression modulus. In contrast to traditional ceramic linear actuators, DEAs show a number of advantages: they are cheap, easy to process, lightweight, flexible and show actuation displacements on a macroscopic scale. ${ }^{6}$ The main reason preventing the widespread use of DEAs is the high electric field necessary to achieve reasonable actuation. ${ }^{7}$ The most common solution to this problem is to increase the relative permittivity of the material by blending it with highly polarizable fillers. ${ }^{8,9,10}$ Although $\varepsilon$ can be significantly increased at concentrations approaching the percolation threshold, dielectric losses increase due to agglomerations and conducting pathways. ${ }^{11}$

The poling of polymers containing dipolar molecules is a well-established method to create polar non-centrosymmetric materials and is frequently used for non-linear optical (NLO) materials. ${ }^{12,13,14}$ The alignment of the dipole in an amorphous polymer is done in a high electric field at temperatures above its glass transition temperature $\left(T_{g}\right)$ followed by freezing of the oriented material by cooling below $T_{g}$ while the electric field is maintained. The oriented dipoles lead to, among other properties, higher $\varepsilon$.

Electroactive Polymer Actuators and Devices (EAPAD) 2015, edited by Yoseph Bar-Cohen, Proc. of SPIE Vol. 9430, 94302U · (c) 2015 SPIE · CCC code: 0277-786X/15/\$18 · doi: 10.1117/12.2085321 
Poleable fillers are a necessary first step towards this goal. They can be realized by synthesizing the particles directly using emulsion polymerization. ${ }^{15,16}$ The feasibility of the method is explored by synthesizing dipoles containing PHEMA particles using miniemulsion polymerization and characterizing their morphology and dielectric response. DR1 and its methacrylate functionalized version (DR1MA) are the most frequently studied dipoles in NLO, and were chosen for this study (Figure 1).

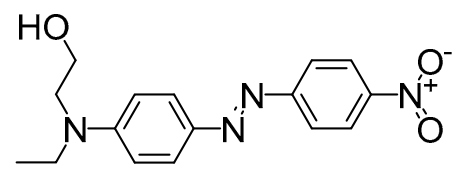

DR1

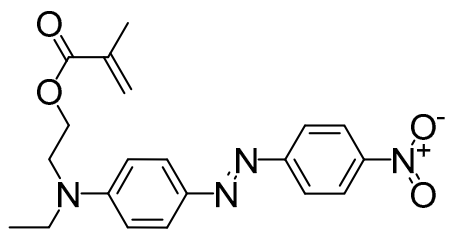

DR1MA

Figure 1. Chemical structure of DR1 and DR1MA.

\section{EXPERIMENTAL PART}

\subsection{Materials}

All chemicals were purchased from Aldrich and used as received, except when noted otherwise. The hydroxyl endterminated PDMS AB116665 $\left(M_{w}=139^{\prime} 000 \mathrm{~g} / \mathrm{mol}\right)$ and the $(25-35 \%$ mehylhydrosiloxane)-dimethylsiloxane-copolymer AB109380 crosslinker (XL) were purchased from ABCR. Solvents were purified and dried by standard procedures. Poly(ethylene-co-butylene)-block-poly(ethylene oxide) surfactant ${ }^{17}$ and DR1MA were synthesized according to literature. $^{18}$

\subsection{Characterization}

${ }^{1} \mathrm{H}$ NMR spectra were recorded on a Bruker Avance-400 spectrometer (400 MHz at room temperature). Gel permeation chromatography (GPC) measurements in tetrahydrofuran (THF) eluent were done with an Agilent 1100/PSS WinGPC 8.1 system calibrated with polymethylmethacrylate standards (Polymer Standards Service). Two columns (100, $1000 \AA$ pore size SDV-Gel) and a flow rate of $1 \mathrm{ml} \mathrm{min}^{-1}$ were used. Signals were detected with a UV and RI detector. GPC measurements with dimethylformamide (DMF) as eluent were done on a GPCv2000 from Waters using a solution of

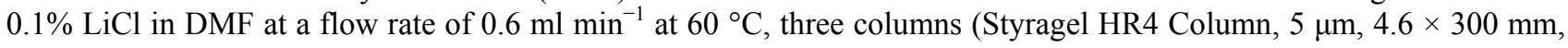
Styragel HR2 Column, $5 \mu \mathrm{m}, 4.6 \times 300 \mathrm{~mm}$, and Styragel HR0.5 Column, $5 \mu \mathrm{m}, 4.6 \times 300 \mathrm{~mm}$ ) and calibrated with polymethylmethacrylate standards. Signals were detected with a RI detector. Thermogravimetric analysis (TGA) was conducted with a Perkin Elmer TGA7 at a heating rate of $20^{\circ} \mathrm{C} \mathrm{min}^{-1}$ under nitrogen gas flow up to $800{ }^{\circ} \mathrm{C}$. Scanning electron microscopy was conducted on a FEI NovaNanoSEM 230 at $10-15 \mathrm{kV}$ acceleration voltage after the samples were sputtered with $7 \mathrm{~nm}$ of platinum. SEM particle size was determined by measuring the diameter of 50 neighboring particles in a representative area. Dynamic light scattering (DLS) was done with a Malvern Zetasized Nano ZS, where the results were averaged from 3 separate measurements. Differential scanning calorimetry (DSC) measurements were done on a Perkin Elmer DSC 8000. The DSC cycle consisted of two heating steps from $20{ }^{\circ} \mathrm{C}$ to $200{ }^{\circ} \mathrm{C}$ and a cooling step from $200{ }^{\circ} \mathrm{C}$ to $20{ }^{\circ} \mathrm{C}$ at a rate of $20^{\circ} \mathrm{C} \mathrm{min}{ }^{-1}$. For the broadband dielectric spectroscopy (BDS) measurements a Novocontrol high impedance Alpha Analyzer was coupled to a Anton Paar MCR rheometer. The rheometer plates $(\varnothing=25$ $\mathrm{mm}$ ), which were electrically isolated from the rest of the device, served as electrodes. The sample height was equivalent to the gap width which was controlled by the rheometer software. This setup allowed measurements of samples in the melt state.

\subsection{Inverse miniemulsion polymerization of PHEMA/Dipole}

A solution of poly(ethylene-co-butylene)-block-poly(ethylene oxide) surfactant and $\alpha, \alpha^{\prime}$ azobisisobutyronitrile (AIBN) in cyclohexane $(\mathrm{CH})$ continuous phase and a solution of DR1 or DR1MA in hydroxyethyl methacrylate (HEMA) dispersed phase were prepared. To dissolve quantities of DR1 or DR1MA above the solubility limit of HEMA, NMP co-solvent was used. For the quantities used, see Table 1 . The two solutions were mixed together and stirred at room temperature to 
obtain a homogenous macroemulsion. The miniemulsion was produced by subjecting the macroemulsion to high shear forces using a Sonics Vibra-cellTM VCX 750 ultrasonicator at $40 \%$ power output (300 watt) for 5 min while being immersed in an iced water bath. The miniemulsion was bubbled through with Ar, and then stirred under inert atmosphere in a preheated bath at $72{ }^{\circ} \mathrm{C}$. After $24 \mathrm{~h}$, the resulting suspension was cooled to room temperature.

Table 1. Preparation of PHEMA using miniemulsion polymerization.

\begin{tabular}{ccccccccc}
\hline \multirow{2}{*}{ Sample } & HEMA & \multicolumn{6}{c}{ Dye } & \multicolumn{6}{c}{ Dye/HEMA NMP CH Surfactant AIBN } \\
\hline & [g] & Type & [mg] & {$[\mathbf{w t} \% \mathbf{c}$} & [g] & [g] & [mg] & {$[\mathbf{m g}]$} \\
\hline A & 1.5 & - & - & 0 & - & 6 & 50 & 100 \\
B1 & 1.5 & DR1 & 20 & 1.3 & - & 6 & 50 & 100 \\
B2 & 0.5 & DR1 & 66 & 11.8 & 0.5 & 8 & 36 & 66 \\
B3 & 0.5 & DR1 & 100 & 16.6 & 0.5 & 8 & 36 & 66 \\
C1 & 1.5 & DR1MA & 20 & 1.3 & - & 6 & 50 & 100 \\
C2 & 0.75 & DR1MA & 50 & 6.3 & - & 6 & 25 & 50 \\
C3 & 0.75 & DR1MA & 100 & 11.8 & - & 6 & 25 & 50 \\
C4 & 0.5 & DR1MA & 133 & 21.0 & 0.25 & 6 & 50 & 25 \\
C5 & 0.5 & DR1MA & 150 & 30 & 0.5 & 8 & 50 & 33 \\
\hline
\end{tabular}

The particles were characterized via SEM, DLS, DSC, TGA and ${ }^{1} \mathrm{H}$ NMR in DMF- $\mathrm{d}_{7}$. DLS measurements were performed on the dispersions which were diluted several times while SEM samples were done by taking a drop of the dispersion and letting it dry on a sample holder. The other measurements were done from filtered samples. Some selected samples were dried at high vacuum and pressed to $25 \mathrm{mg}$ pellets in order to measure their impedance with the rheometer setup. The pellets were heated to $195^{\circ} \mathrm{C}$, well above the $T_{g}$ of the samples, upon which the isolated rheometer plate was lowered to contact the sample in melt state at a determined sample height. Impedance spectra were obtained at $10{ }^{\circ} \mathrm{C}$ intervals from $195^{\circ} \mathrm{C}$ to $25^{\circ} \mathrm{C}$. The measurement was deemed acceptable when the sample was found in a monolithic, bubble-free state after the removal of the rheometer plate.

\subsection{Composites of PHEMA/Dipole particles in PDMS}

PDMS composites with $50 \mathrm{wt} \%$ of selected particles were produced by adding the PDMS matrix and (25-35\% mehyl hydrosiloxane)-dimethylsiloxane-copolymer crosslinker (XL) to a dispersion of particles in $\mathrm{CH}$ under stirring (see Table 2). When a homogeneous dispersion was formed, dibutyltin dilaurate (DBTL) catalyst was added and films were then produced with the doctor blade method using a Zehntner ZUA 2000 set at $300 \mu \mathrm{m}$ gap height. After drying the films at $80^{\circ} \mathrm{C}$ for several days, thin films with thicknesses around $100 \mu \mathrm{m}$ were obtained. The dispersion quality of the composites was verified by freeze-breaking them in liquid nitrogen and examining the platinum sputtered fracture surface via SEM.

Table 2. Preparation of HEMA/PDMS composite films.

\begin{tabular}{cccccccc}
\hline \multirow{2}{*}{ Sample } & \multicolumn{2}{c}{ Particle } & \multicolumn{2}{c}{ PDMS } & XL & DBTL & CH \\
\cline { 2 - 7 } & Type & {$[\mathbf{g}]$} & {$[\mathbf{w t} \%]$} & {$[\mathbf{g}]$} & {$[\boldsymbol{\mu l}]$} & {$[\boldsymbol{\mu l}]$} & {$[\mathbf{g}]$} \\
\hline C-A & A & 0.5 & 50 & 0.5 & 50 & 10 & 3 \\
C-C3 & C3 & 0.5 & 50 & 0.5 & 50 & 10 & 3 \\
\hline
\end{tabular}

\section{RESULTS AND DISCUSSION}

\subsection{PHEMA/Dipole particles: composition and particle size determination}

The maximum dipole concentration in the particles is generally limited by their solubility in the dispersed phase. As such, DR1 could only be incorporated at $1.3 \mathrm{wt} \%$. While it has been possible to significantly increase the DR 1 content in the miniemulsion from $1.3 \mathrm{wt} \%$ to $11.8 \mathrm{wt} \%$ when using NMP co-solvent, a further increase in the dipole content lead to 
large flakes in the miniemulsion after polymerization. By using a methacrylate-functionalized DR1 the solubility of the dipole could also be increased to $11.8 \mathrm{wt} \%$. As can be seen in the SEM images (Figure 2) the polymerization produced particles in the sub-micrometer range. Their sizes according to the SEM images and DLS number size distribution are noted in Table 3. Again the DR1MA content can be raised to $21 \mathrm{wt} \%$ with NMP co-solvent, above which the emulsion becomes unstable over the time-period of the polymerization. While the dipole content could possibly be further optimized by changing the nature and the amount of co-solvent, this was not further attempted.

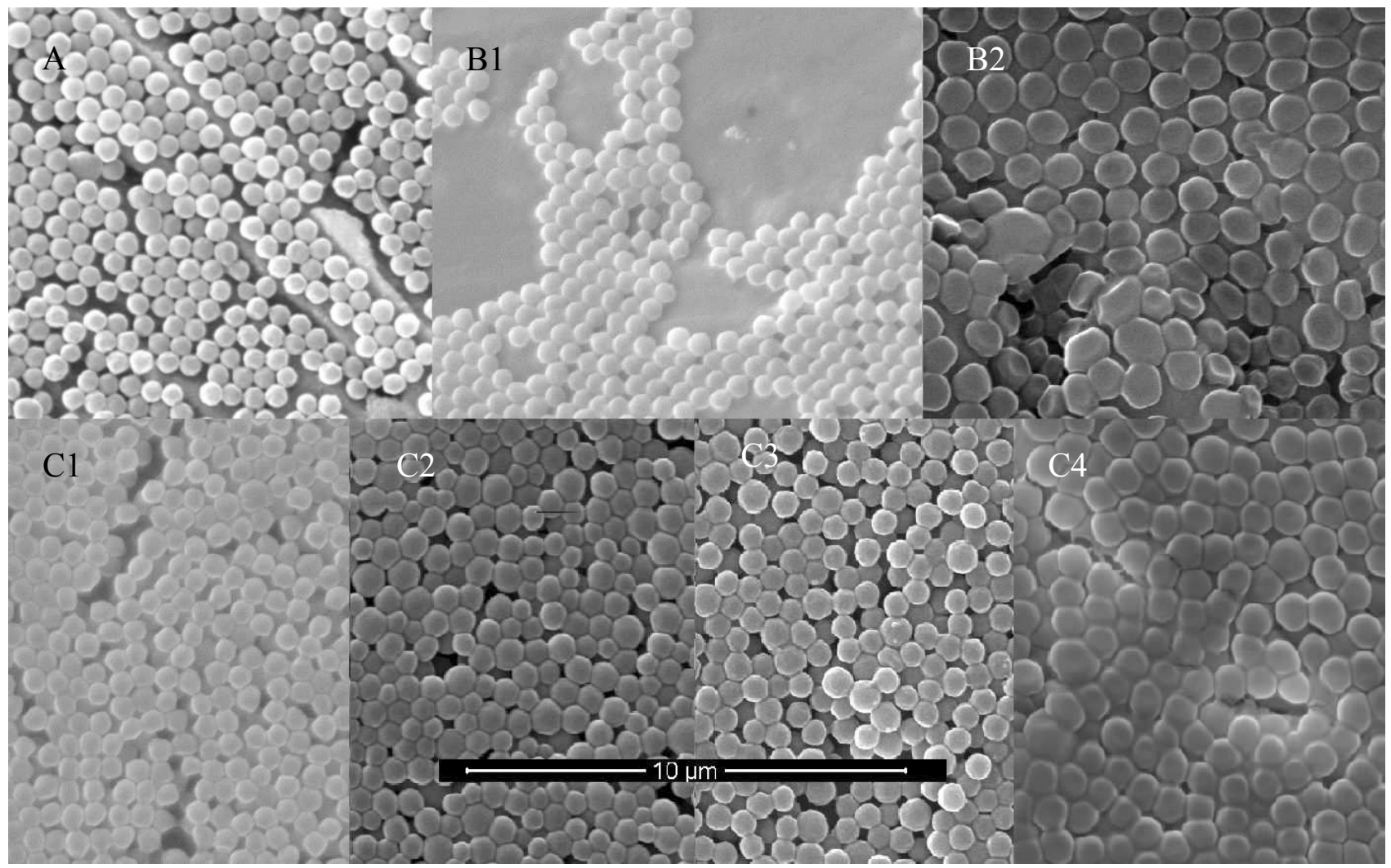

Figure 2. SEM images of PHEMA particles containing dipoles.

It was found that spherical particles were obtained when encapsulating DR1 without NMP. In contrast, the particles produced with a co-solvent, while still roughly spherical, have a less uniform shape and are flattened against the substrate in the SEM image. This make them seem larger $(820 \mathrm{~nm})$ than compared to the particles produced without cosolvent $(480 \mathrm{~nm})$, but DLS shows that they are of roughly the same size when in solution. A systematic investigation of the influence of the dipole on the particle size was not carried out, but it seems from the data in Table 2 as if the dipole has no noteworthy effect on such.

The NMR spectra (Figure 3) were recorded using particles obtained by filtering the dispersion and subsequently washing the particles with $\mathrm{CH}$. The spectra confirm the formation of PHEMA and PHEMA-DR1MA copolymer, as well as the presence of DR1. The amount of dipole incorporated into the particles corresponds to the amount in the miniemulsion prior to the polymerization. The molecular weight and the PDI of the prepared polymers can be found in Table 3 . The PHEMA sample A has a molecular weight of $95^{\prime} 000 \mathrm{~g} / \mathrm{mol}$ and a polydispersity index (PDI) of 2.1. The addition of dipoles generally causes the PDI to increase, up to a PDI of 8.4 for sample C4. Data on the thermal transitions and stability of the samples A and C1 to C4 can be found in Table 4 as determined by DSC and TGA. The low thermal stability of $\mathrm{C} 4$ can be attributed to the presence of NMP.

Due to the limited amount of DR1 which can be encapsulated in PHEMA, even with a co-solvent, it was deemed best to focus future efforts on the copolymer system. 
The particles form stable dispersions in $\mathrm{CH}$ directly after the polymerization. By using these dispersions directly to produce composites in PDMS, agglomeration of the particles was avoided. This is evident by the homogeneous particle distribution within the composites C-A and C-C3 (Figure 4).

Table 3. Size and GPC results for the PHEMA samples.

\begin{tabular}{ccccc}
\hline \multirow{2}{*}{ Sample } & \multicolumn{2}{c}{ Particle diameter } & $\mathbf{M}_{\mathbf{n}}$ & \multirow{2}{*}{ PDI } \\
\cline { 2 - 5 } & SEM [nm] & DLS [nm] & {$\left[\mathbf{k g ~ m o l}^{-\mathbf{1}}\right]$} & \\
\hline $\mathrm{A}$ & $570 \pm 80$ & $650 \pm 80$ & 95 & 2.1 \\
$\mathrm{~B} 1$ & $480 \pm 40$ & $510 \pm 200$ & 193 & 4.2 \\
$\mathrm{~B} 2$ & $820 \pm 110$ & $540 \pm 170$ & 92 & 2.1 \\
$\mathrm{~B} 3$ & $\mathrm{~N} / \mathrm{A}$ & $\mathrm{N} / \mathrm{A}$ & - & - \\
$\mathrm{C} 1$ & $480 \pm 50$ & $560 \pm 180$ & 186 & 4.0 \\
$\mathrm{C} 2$ & $550 \pm 100$ & $520 \pm 90$ & 105 & 5.7 \\
$\mathrm{C} 3$ & $570 \pm 70$ & $660 \pm 130$ & 96 & 5.5 \\
$\mathrm{C} 4$ & $750 \pm 90$ & $560 \pm 100$ & 69 & 8.4 \\
$\mathrm{C} 5$ & N/A & N/A & - & - \\
\hline
\end{tabular}

Table 4. Glass transition temperature $\left(T_{g}\right)$ and decomposition temperature $\left(T_{d}\right)$ as determined by DSC and TGA.

\begin{tabular}{|c|c|c|}
\hline \multicolumn{3}{|c|}{ Sample $T_{g}\left[{ }^{\circ} \mathbf{C}\right] T_{\mathbf{d}}\left[{ }^{\circ} \mathbf{C}\right]$} \\
\hline A & 96 & 415 \\
\hline $\mathrm{C} 1$ & 93 & 380 \\
\hline $\mathrm{C} 2$ & 96 & 350 \\
\hline $\mathrm{C} 3$ & 92 & 335 \\
\hline $\mathrm{C} 4$ & 65 & 290 \\
\hline
\end{tabular}

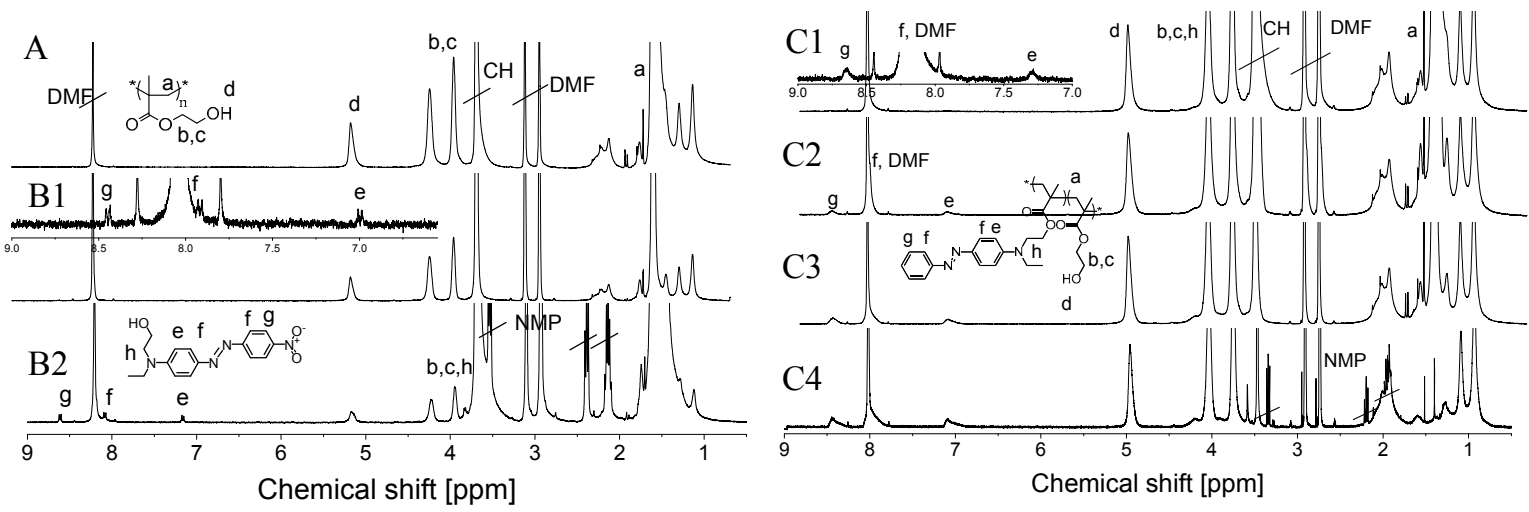

Figure $3 .{ }^{1} \mathrm{H}$ NMR spectra of the PHEMA particles without (A) and with DR1 dipoles (B1, B2) and of the PHEMA-coDR1MA particles (C1-C4) produced by miniemulsion polymerization. 


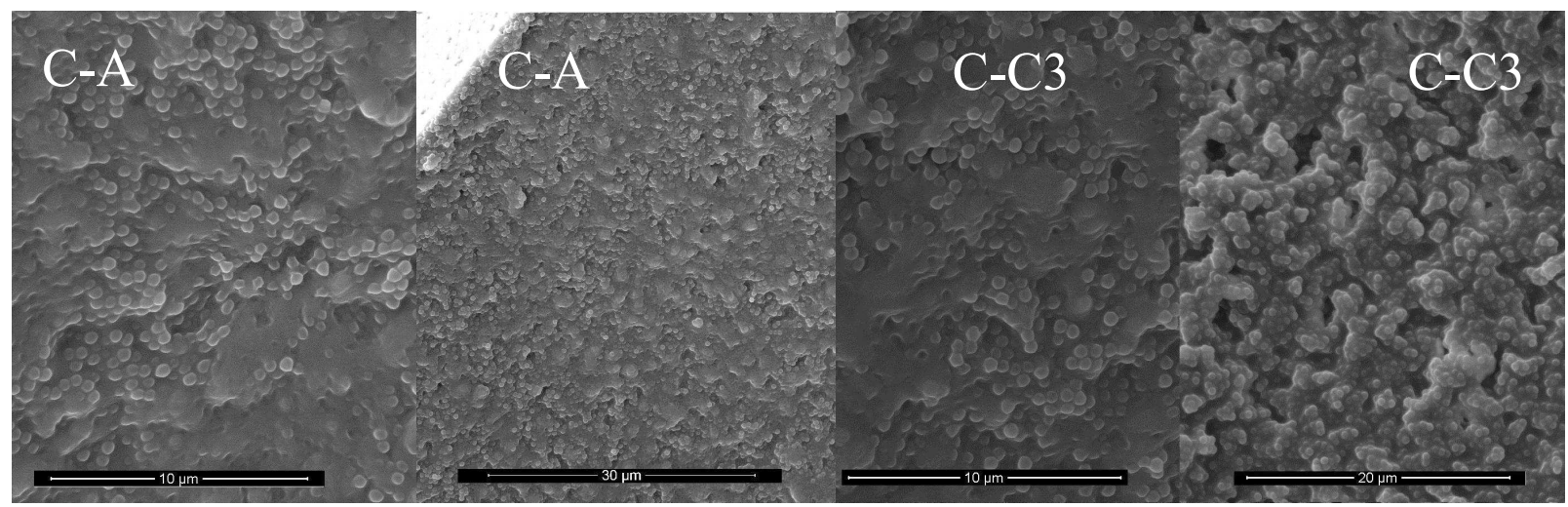

Figure 4. SEM images of the composites C-A and C-C3

\subsection{PHEMA/Dipole particles: BDS-analysis}

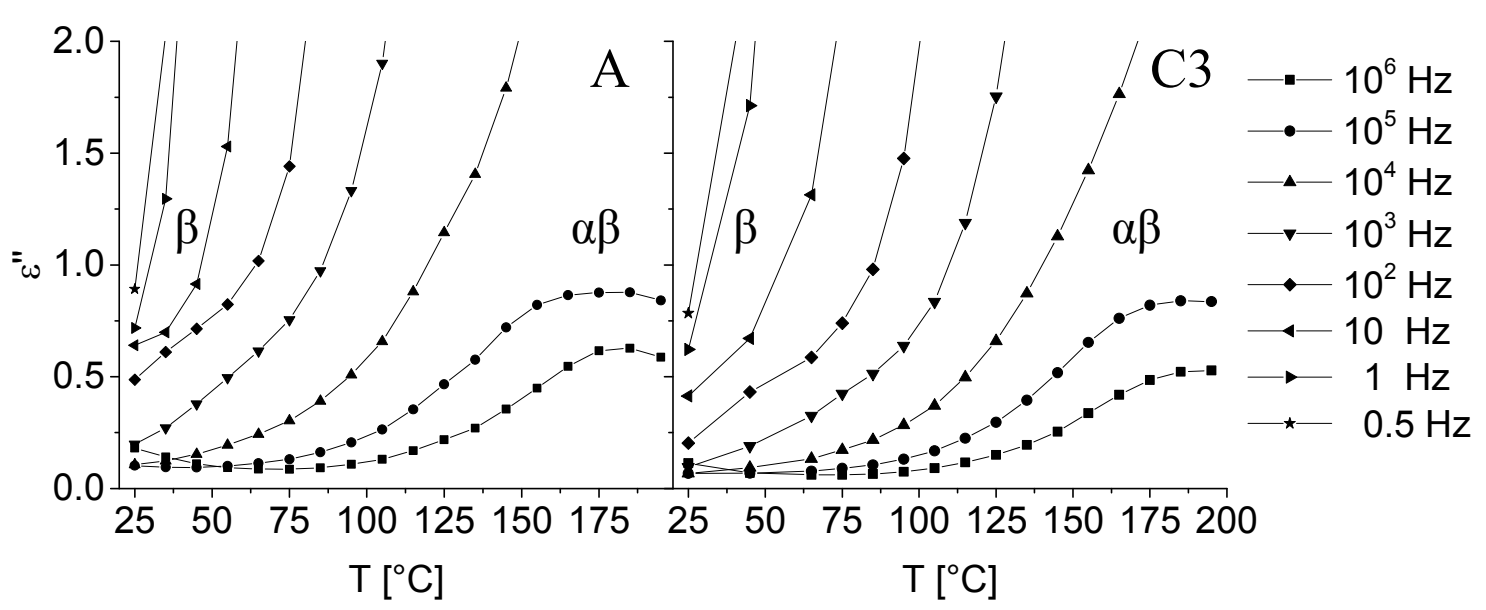

Figure 5. Imaginary permittivity ( $\left.\varepsilon^{\prime \prime}\right)$ versus temperature for samples A and C3 as measured by BDS.

Impedance measurements (Figure 5) were done on PHEMA samples (A) and on PHEMA-co-DR1MA samples with the maximum dipole content produced without co-solvent (C3). Data for sample A reveals a relaxation behavior identical to the reported behavior of bulk PHEMA. ${ }^{19,20}$ The surfactant therefore has no influence on the relaxation behavior of the polymer. The spectrum is dominated by a rapidly increasing $\varepsilon$ " signal at low frequencies $\left(0.5-10^{4} \mathrm{~Hz}\right)$ which is mirrored by a rapid increase in $\varepsilon^{\prime}$. This behavior is typically attributed to electrode polarization due to conductivity contributions of the sample. A single peak is visible at high frequencies $\left(10^{5}-10^{6} \mathrm{~Hz}\right)$ which was speculated to be either a $\beta$-relaxation peak or the $\alpha \beta$-merge peak. ${ }^{19}$ A shoulder to the electrode polarization contribution is visible at lower frequencies $(1-100$ $\mathrm{Hz}$ ) which was attributed to the $\beta$ relaxation. ${ }^{20}$ These assertions can be partially substantiated by analyzing the data with the phenomenological Havriliak-Negami (HN) function, ${ }^{21} \varepsilon^{*}=\varepsilon_{\infty}+i \frac{\sigma_{d c}}{\varepsilon_{0} \omega}+\sum \frac{\Delta \varepsilon_{n}}{\left\{1+\left[i \omega \tau_{n}\right]^{1-\alpha_{n}}\right\}^{\beta_{n}}}$, where $\varepsilon^{*}$ is the complex impedance, $\varepsilon_{\infty}$ the high frequency permittivity, $\sigma_{d c}$ the dc-conductivity, $\varepsilon_{0}$ the vacuum permittivity. $\Delta \varepsilon_{n}$ and $\tau_{n}$ are the relaxation strength and mean relaxation time, the parameters $\alpha_{n}$ and $\beta_{n}$ describe the width and asymmetry of the dielectric loss peak $\varepsilon^{\prime}$ " of process $\mathrm{n}$. The conductivity contribution to $\varepsilon^{*}$ is relatively important here as the $\beta$ - and the $\alpha \beta$-merge peak both merge with the dc contributed part. Unfortunately the spectra for $\mathrm{C} 3$ at temperatures where the $\alpha \beta$-merge peak is relevant were not resolved enough to apply the HN-fit. 


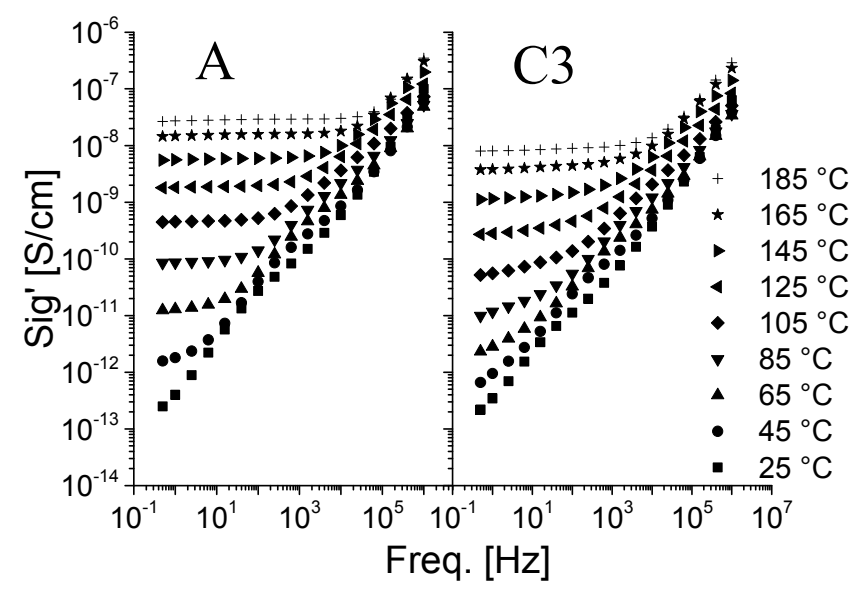

Figure 6. Real conductivity part of samples A and C3 at different frequencies as measured by BDS.

As a matter of practicality, $\sigma_{d c}$ was taken as the conductivity at $0.5 \mathrm{~Hz}$, the lowest measured frequency. At temperatures below $100{ }^{\circ} \mathrm{C}$ that value was slightly different from $\sigma_{d c}$, as the conductivity was showing the beginnings, but has not reached a steady state yet (Figure 6). It follows that in order to fit data taken at temperatures below $100{ }^{\circ} \mathrm{C}$ the high frequency part of the spectra was given more weight, as can be seen representatively in Figure 7 . Above $100{ }^{\circ} \mathrm{C}, \sigma_{d c}$ could accurately be measured by the conductivity value at $0.5 \mathrm{~Hz}$ as it has reached a steady state.
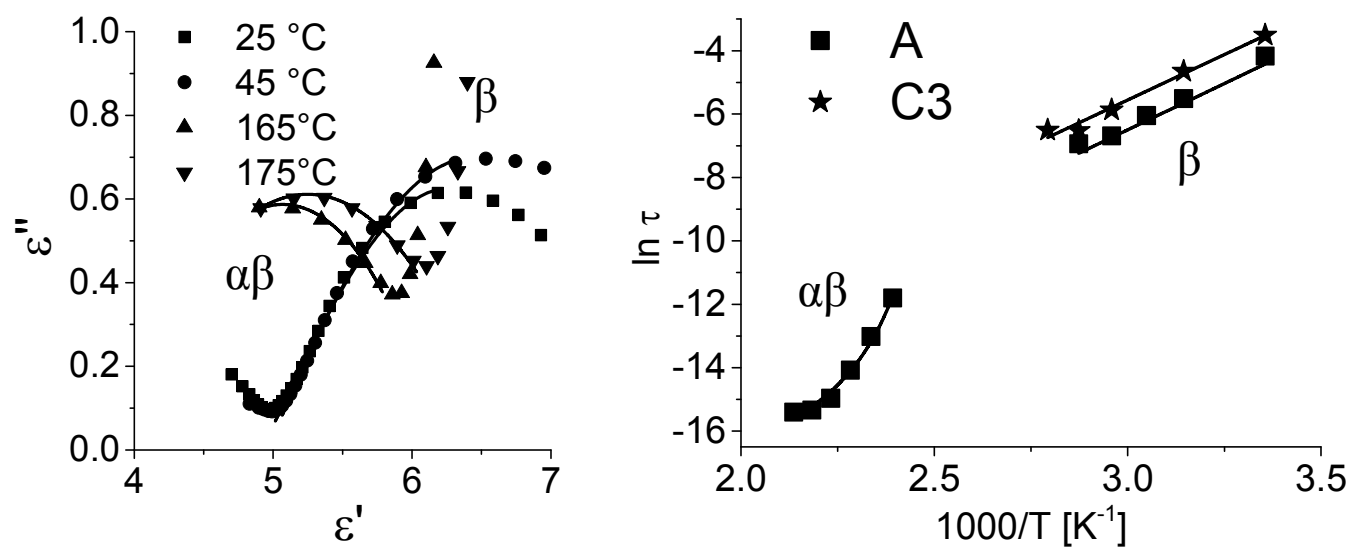

Figure 7. Cole-cole plot of four representative relaxation curves of sample A (left) from which the dc-contribution has been subtracted (scatter plot) and their fit-curves according to the HN-function (line-plot) and the temperature dependent mean relaxation time $\tau_{n}$ extracted from the fitted $\mathrm{HN}$-functions for samples $\mathrm{A}$ and $\mathrm{C} 3$ (right).

The $\beta$ relaxation hinted at in the imaginary permittivity plot can be quantified after extracting the fit-parameters. The evolution of $\tau$ with temperature (figure 7) suggests an Arrhenius-type relaxation process typically associated with sidechain movements. The activation energy for this process has been calculated to be $48.4 \pm 5.8 \mathrm{~kJ} / \mathrm{mol} \mathrm{in}$ sample A and $47.4 \pm 3.7 \mathrm{~kJ} / \mathrm{mol}$ in sample $\mathrm{C} 3$, which indicates that the $\beta(\mathrm{A})$ and $\beta(\mathrm{C} 3)$ can be assigned to the same side-chain relaxation process. Unfortunately the relaxation peak corresponding to the relaxation of the dipole was not resolved in the measured range of the current experiment. 


\section{CONCLUSIONS}

DR1 dipole was successfully incorporated into PHEMA particles using miniemulsion polymerization. The maximum dipole concentration is limited by the solubility of the dipole in the dispersed phase. The concentration of the dipole can be increased by either chemically modifying the dipole or using a co-solvent in the dispersed phase. The incorporation of the dipole was confirmed with ${ }^{1} \mathrm{H}$ NMR and matched mostly with the target concentration. Particle size distributions were reasonably narrow, while the inclusion of the dipole and the use of a co-solvent don't seem to influence the particle size. BDS analysis reveals that particles produced in such a manner do not exhibit any additional relaxation phenomena. The $\beta$ relaxation of the hydroxyethyl-side chain was revealed and quantified in both the pure PHEMA sample and the sample containing dipoles. The $\alpha \beta$-merge peak was observed in both samples but could only be quantified for the pure PHEMA sample. Unfortunately the relaxation of the dipole was not resolved in the measurement range, due to the typically large conductivity-contribution in PHEMA. It has been shown that the relaxation phenomena in PHEMA do not change significantly when being produced by miniemulsion polymerization and when dipoles are incorporated.

The polymerized particles form stable dispersions in cyclohexane which allow formation of homogeneous PHEMAPDMS composites. Further investigations into the relaxation behavior of the particles and the composite are needed; especially the dipole relaxation has to be identified, if the system is to be developed further.

\section{REFERENCES}

[1] Nahata, A., Shan, J. H., Yardley, J. T., Wu, C. J., "Electrooptic Determination of the Nonlinear-Optical Properties of Acovalently Functionalized Disperse Red-1 Copolymer," J. Opt. Soc. Am. B, 10(9), 1553-1564 (1993).

[2] Pelrine, R. E.; Kornbluh, R. D.; Joseph, J. P., "Electrostriction of polymer dielectrics with compliant electrodes as a means of actuation," Sensor Actuat. a-Phys., 64(1), 77-85 (1998).

[3] Ashley, S., “Artificial Muscles,” Scientific American, 289(4), 52-59 (2003).

[4] Carpi, F.; De Rossi, D.; Kornbluh, R.; Pelrine, R.; Sommer-Larsen, P., [Dielectric Elastomers as Electromechanical Transducers: Fundamentals, Materials, Devices, Models and Applications of an Emerging Electroactive Polymer Technology], Elsevier, New York, ix-xiii (2008).

[5] Pelrine, R.; Kornbluh, R.; Pei, Q. B.; Joseph, J., "High-speed electrically actuated elastomers with strain greater than $100 \%$," Science, 287(5454), 836-839 (2000).

[6] Bar-Cohen, Y., "EAP as artifical muscles - Progress and challenges," Proc. SPIE 5385, 10-16 (2004).

[7] Kofod, G., "The static actuation of dielectric elastomer actuators: how does pre-stretch improve actuation?" Journal of Physics D: Applied Physics, 41(21), 215405 (2008).

[8] Carpi, F.; De Rossi, D., "Improvement of electromechanical actuating performances of a silicone dielectric elastomer by dispersion of titanium dioxide powder," IEEE Transactions on Dielectrics and Electrical Insulation, 12(4), 835-843 (2005).

[9] Bar-Cohen, Y.; Egede Daugaard, A.; Hassouneh, S. S.; Kostrzewska, M.; Bejenariu, A. G.; Skov, A. L., "Highdielectric permittivity elastomers from well-dispersed expanded graphite in low concentrations," Proc. SPIE 8687, 868729-868729-11 (2013).

[10] Yang, D.; Tian, M.; Li, D.; Wang, W.; Ge, F.; Zhang, L., "Enhanced dielectric properties and actuated strain of elastomer composites with dopamine-induced surface functionalization," Journal of Materials Chemistry A, 1(39), 12276 (2013).

[11]Leng, J.; Stoyanov, H.; Kollosche, M.; McCarthy, D.; Becker, A.; Risse, S.; Kofod, G.; Asundi, A. K.; Ecke, W., "Sub-percolative composites for dielectric elastomer actuators," Proc. SPIE 7493, 74930Q-74930Q-8 (2009).

[12] Purbrick, M. D., "Organic materials for non-linear optics," Polymer International, 30(2), 284-284 (1993)

[13]Bauer, S., "Poled polymers for sensors and photonic applications," Journal of Applied Physics, 80(10), 55315558 (1996)

[14] Hoffman, R. C.; Pritchett, T. M.; Orlicki, J. A.; Dougherty, J. M.; Lambeth, R. H.; Rawlett, A. M.; Herman, W. N.; Park, D. H., "High-Voltage Poling of Bulk Guest-Host Polymers," Acs. Sym. Ser., 1039, 97-109 (2010).

[15]Landfester, K., "Polyreactions in miniemulsions," Macromol. Rapid Comm., 22(12), 896-936 (2001)

[16] Schork, F. J.; Luo, Y.; Smulders, W.; Russum, J. P.; Butté, A.; Fontenot, K., [Miniemulsion Polymerization], Springer, New York, 129-255 (2005). 
[17] Ko, Y. S., Circu, M. V., Geiger, T., Dunki, S., Nuesch, F. A., Opris, D. M., „Synthesis of poly(ethylene-cobutylene)-block-poly(ethylene oxide) surfactant and its use in the synthesis of polyhydroxyethyl methacrylate nanoparticles containing azo-dye," Rsc Advances, 4(66), 35027-35034 (2014).

[18] Nahata, A., Shan, J. H., Yardley, J. T., Wu, C. J., "Electrooptic Determination of the Nonlinear-Optical Properties of Acovalently Functionalized Disperse Red-1 Copolymer," J. Opt. Soc. Am. B, 10(9), 1553-1564 (1993).

[19] Mohomed, K., Gerasimov, T. G., Moussy, F., Harmon, J. P., "A broad spectrum analysis of the dielectric properties of poly(2-hydroxyethyl methacrylate)," Polymer, 46(11), 3847-3855 (2005).

[20] Ribelles, J. L. G., Calleja, R. D., "Effect of the Cooling Rate in the Formation of Glass on the AlphaRelaxations and Beta-Relaxations of Some Amorphous Polymers," Polym. Eng. Sci., 24(15), 1202-1204, (1984).

[21] Havriliak, S.; Negami, S., "A Complex Plane Representation of Dielectric and Mechanical Relaxation Processes in Some Polymers," Polymer, 8(4), 161-210 (1967). 